Journal of

Synchrotron

Radiation

ISSN 0909-0495

Received 31 July 2008

Accepted 19 February 2009

\title{
100 ps time-resolved solution scattering utilizing a wide-bandwidth X-ray beam from multilayer optics
}

\author{
K. Ichiyanagi, ${ }^{a *}$ T. Sato, ${ }^{a, b}$ S. Nozawa, ${ }^{a}$ K. H. Kim, ${ }^{c}$ J. H. Lee, ${ }^{c}$ J. Choi, ${ }^{c}$ A. Tomita, ${ }^{a, b}$ \\ H. Ichikawa, ${ }^{a}$ S. Adachi, ${ }^{a, d}$ H. Ihee ${ }^{\mathrm{c} *}$ and S. Koshihara ${ }^{\mathrm{a}, \mathrm{b}}$

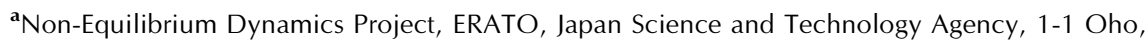 \\ Tsukuba, Ibaraki 305-0801, Japan, '⿳亠丷厂 Tokyo Institute of Technology, 2-12-1 Oh-okayama, \\ Megro-ku, Tokyo 152-8551, Japan, 'Center for Time-Resolved Diffraction, Department of \\ Chemistry, KAIST, Daejeon 305-701, Republic of Korea, and ${ }^{\mathbf{d} H i g h}$ Energy Accelerator Research \\ Organization, 1-1 Oho, Tsukuba, Ibaraki 305-0801, Japan. E-mail: ichiyana@post.kek.jp, \\ hyotcherl.ihee@kaist.ac.kr
}

\begin{abstract}
100 ps time-resolved X-ray solution-scattering capabilities have been developed using multilayer optics at the beamline NW14A, Photon Factory Advanced Ring, KEK. X-ray pulses with an energy bandwidth of $\Delta E / E=1-5 \%$ are generated by reflecting $\mathrm{X}$-ray pulses $(\Delta E / E=15 \%)$ through multilayer optics, made of $\mathrm{W} / \mathrm{B}_{4} \mathrm{C}$ or depth-graded $\mathrm{Ru} / \mathrm{C}$ on silicon substrate. This tailor-made wide-bandwidth X-ray pulse provides high-quality solution-scattering data for obtaining photo-induced molecular reaction dynamics. The time-resolved solution scattering of $\mathrm{CH}_{2} \mathrm{I}_{2}$ in methanol is demonstrated as a typical example.
\end{abstract}

Keywords: time-resolved solution scattering; photodissociation reaction; liquidography; multilayers.

\section{Introduction}

Studying photo-induced reactions in the solution phase with subnanosecond time-resolution offers opportunities for understanding fundamental molecular reaction dynamics in chemistry and biology. Time-resolved X-ray diffraction using 100 ps X-ray pulses from a synchrotron source can elucidate the molecular geometry involved in photo-induced reaction pathways (Plech et al., 2004; Ihee et al., 2005; Georgiou et al., 2006; Davidsson et al., 2005; Kim et al., 2006; Lee et al., 2006, $2008 a$,b; Kong et al., 2007, 2008). An X-ray pulse with $\sim 3 \%$ energy bandwidth has been used for solution-scattering experiments at the ID09B beamline of the European Synchrotron Radiation Facility (Plech et al., 2002, 2004; Wulff et al., 2004, 2006; Mirloup et al., 2004; Ihee, 2009). Significant improvements in the signal-to-noise ratios of the experimental data have been reported for photochemical reactions of halogen compounds in solution. For example, the structural dynamics of $\mathrm{C}_{2} \mathrm{H}_{4} \mathrm{I}_{2}$ in methanol were studied using the highflux X-ray pulse at the ID09B beamline (Ihee et al., 2005), and the reaction pathways and associated transient molecular structures in solution were resolved by the combination of theoretical calculations and global fitting analysis (Lee et al., 2006; Cammarata et al., 2006).

Recently, beamline NW14A at PF-AR, KEK, was constructed as a $100 \mathrm{ps}$ time-resolved X-ray beamline (Nozawa et al., 2007) using monochromatic or white X-rays. Its high-flux white $\mathrm{X}$-rays have $\Delta E / E \simeq 15 \%$ energy bandwidth when an undulator of period length $20 \mathrm{~mm}$ is used. To check the feasibility of time-resolved scattering with such a wide bandwidth and to search for the optimal bandwidth, we simulated the Debye scattering curves for the reaction $\mathrm{C}_{2} \mathrm{H}_{4} \mathrm{I}_{2}$ $\rightarrow \mathrm{C}_{2} \mathrm{H}_{4} \mathrm{I}+\mathrm{I}$ using (i) a $15 \%$ bandwidth with the default X-ray energy distribution for the undulator spectrum on NW14A, (ii) a Gaussian spectrum with $5 \%$ energy bandwidth, (iii) a Gaussian spectrum with a $1 \%$ energy bandwidth, and (iv) a Gaussian spectrum with $0.01 \%$ energy bandwidth, as shown in Fig. 1. The photon flux of the X-ray pulse increases with the energy bandwidth, but the simulation shows that the $15 \%$ energy bandwidth with the default spectrum with a long tail is not suitable for time-resolved solution-scattering experiments owing to insufficient $q$-resolution. The long tail of the default $\mathrm{X}$-ray spectrum induces a much higher extent of blurring at high scattering angles than a symmetric Gaussian spectrum with the same bandwidth. For this reason, the X-ray spectrum with a long tail at ID09B of ESRF with $\sim 3 \%$ bandwidth is comparable with a Gaussian spectrum with $\sim 10 \%$ bandwidth. In contrast, when we compare the calculated scattering curve using the Gaussian spectrum with $1 \%$ and $5 \%$ energy bandwidth X-rays with that with a $0.01 \%$ energy bandwidth, three calculated curves seem to reproduce the same quality. In addition, the total flux of the $5 \%$ energy bandwidth X-ray beam will be higher than that of the monochromatic X-rays ( $\sim 0.01 \%$ energy bandwidth) from a $\mathrm{Si}$ single crystal by a factor of 250 . The total flux of the $5 \%$ energy bandwidth $\mathrm{X}$-rays is about five times more than that of the $1 \%$ energy bandwidth X-rays. Therefore, the data collecting time using the $5 \%$ energy bandwidth X-rays becomes shorter than when 
Figure 1

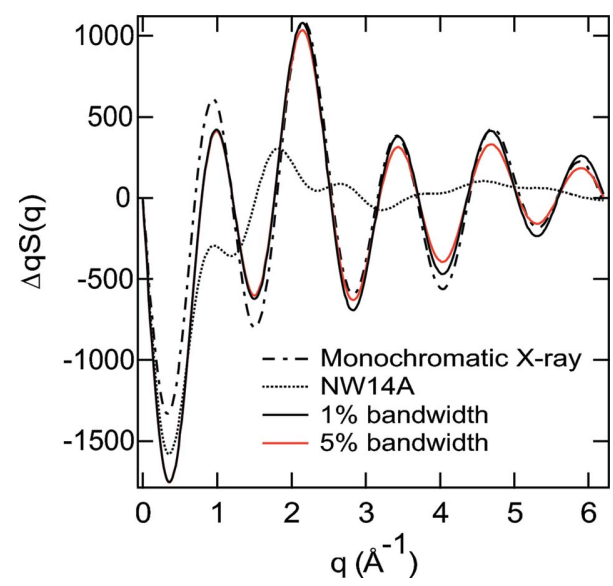

Debye scattering curves calculated for the model reaction $\mathrm{C}_{2} \mathrm{H}_{4} \mathrm{I}_{2} \rightarrow$ $\mathrm{C}_{2} \mathrm{H}_{4} \mathrm{I}+\mathrm{I}$ using a $0.01 \%$ (monochromatic) Gaussian X-ray energy profile (dot-dashed line), 5\% Gaussian X-ray energy profile (red line), 1\% Gaussian X-ray energy profile (solid line), and $15 \%$ default X-ray energy profile with a long tail (dotted line).

using the monochromatic $\mathrm{X}$-rays and the $1 \%$ energy bandwidth $\mathrm{X}$-rays. These estimations clearly indicate that the preparation of X-ray pulses with $\Delta E / E \simeq 5 \%$ has a very significant merit for promoting a time-resolved $\mathrm{X}$-ray solutionscattering experiment, and, thus, prompted us to reduced the bandwidth from the default $15 \%$ down to less than the $\sim 5 \%$ energy bandwidth of multilayer optics.

In our experimental set-up, the multilayer optics can produce $\mathrm{X}$-rays with a $1-5 \%$ energy bandwidth, and allow us to measure the time-resolved solution-scattering with the undulator at the NW14A beamline. The purpose of this paper is to present a detailed account of achievements with the multilayer optics. We succeeded in collecting high-quality time-resolved solution-scattering data for the $\mathrm{CH}_{2} \mathrm{I}_{2}$ photo- chemical reaction in methanol and briefly report the experimental aspects.

\section{Experimental set-up}

A schematic diagram of the experimental set-up is shown in Fig. 2. The experimental system consists of an amplified Ti:sapphire laser system for providing laser pulses to excite the liquid sample, an X-ray pulse selector (XPS) to select single X-ray pulses, a heat-load chopper (Gembicky et al., 2007), laser and X-ray shutters, and a sapphire nozzle to provide a stable liquid jet. This beamline gives a white $\mathrm{X}$-ray pulse in the energy range 13-18 keV using an undulator with a period length of $20 \mathrm{~mm}$ at a repetition rate of $794 \mathrm{kHz}$ and with a pulse duration of about 100 ps. The scattered images were recorded on an integrating charge-coupled device detector (MarCCD165, MarUSA) of diameter $165 \mathrm{~mm}$. Details of the set-up have been described elsewhere (Nozawa et al., 2007).

\section{Production of a wide-bandwidth X-ray beam using multilayer optics}

We have utilized two types of multilayer optics. The first one is $\mathrm{W} / \mathrm{B}_{4} \mathrm{C}(d=27.7 \AA$, X-ray Company, Russia) on a Si single crystal with a size of $50 \times 50 \times 5 \mathrm{~mm}$, which provides an X-ray spectrum with $\sim 1 \%$ energy bandwidth and in which the peak energy of the X-ray spectrum can be changed by tilting the angle of the multilayer optics, as shown in Fig. 3(a). The second multilayer, which is a depth-graded $\mathrm{Ru} / \mathrm{C}$ layer $(d=$ $40 \AA$ A , NTT Advanced Technology, Japan), produces a $\sim 5 \%$ energy bandwidth from the undulator spectrum, as shown in Fig. 3(b). A real image of the multilayer mirror installed in the

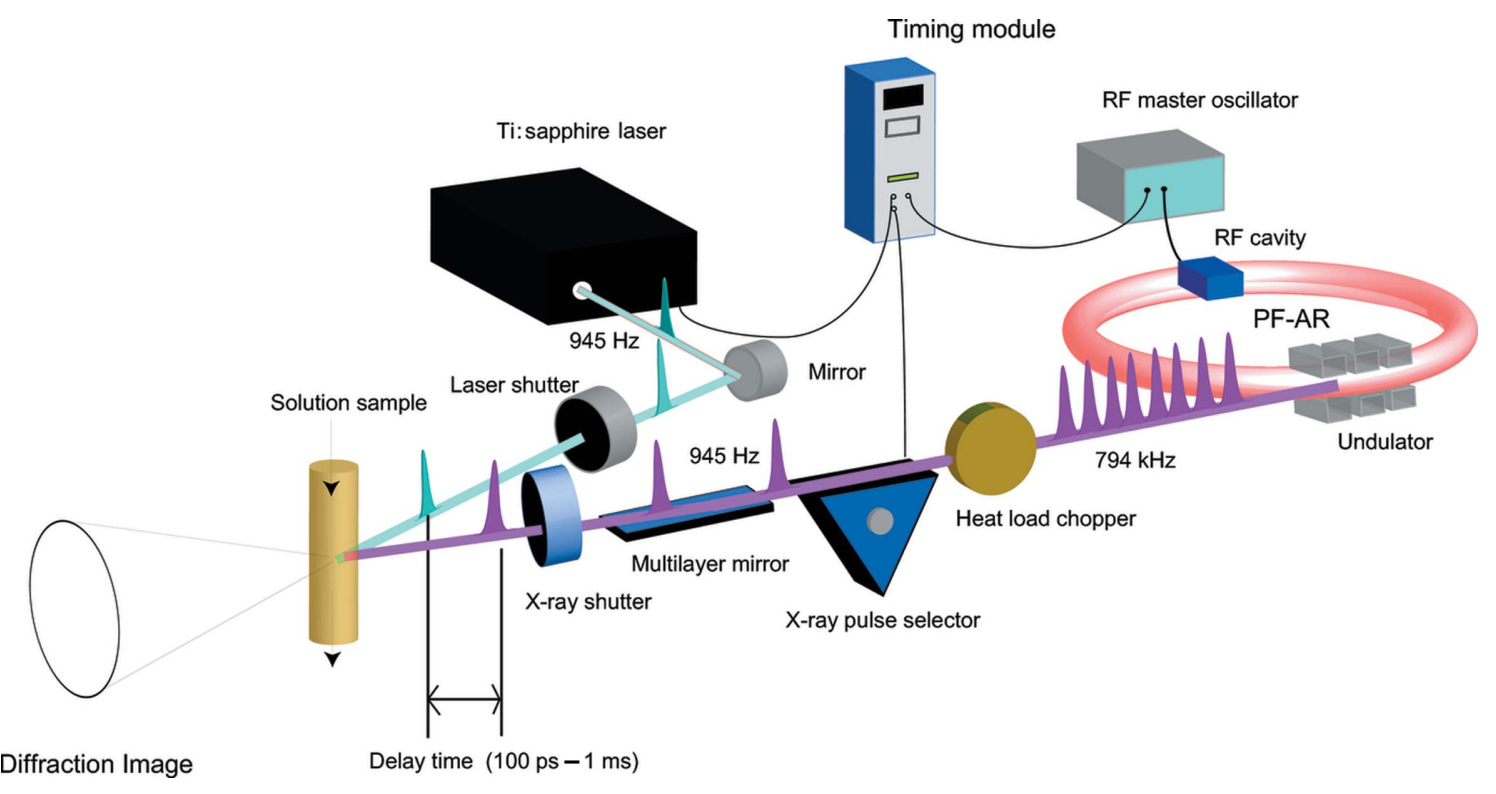

Figure 2

Schematic diagram of the time-resolved solution X-ray scattering at beamline NW14A, PF-AR. The wide-bandwidth $(\Delta E / E=1-5 \%) \mathrm{X}$-ray pulses at $945 \mathrm{~Hz}$ are provided from the multilayer optics downstream of the X-ray pulse selector. The laser and the X-ray pulse selector are synchronized by using the RF master oscillator. 

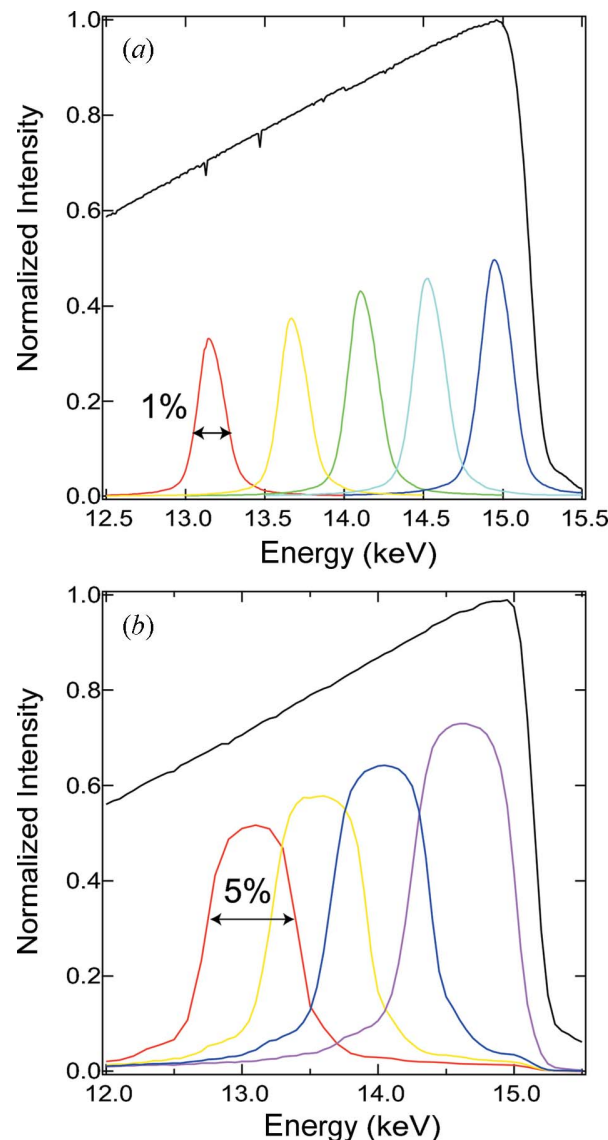

Figure 3

Wide-bandwidth X-ray pulses were produced by multilayer optics from the undulator spectrum. The peak energy position is controlled by changing the incident angle. The black curve is the $\mathrm{X}$-ray spectrum from the undulator, with a gap of $11 \mathrm{~mm}$. (a) X-ray spectra using the W/B $\mathrm{B}_{4} \mathrm{C}$ multilayer optics. The X-ray bandwidth is about $1 \%$. (b) X-ray spectra using the depth-graded $\mathrm{Ru} / \mathrm{C}$ multilayer optics. The X-ray bandwidth is $5 \%$.

vacuum chamber is shown in Fig. 4. The diameter of the vacuum chamber placed on a swivel stage is $160 \mathrm{~mm}$. The multilayer optics is mounted on a water-cooled copper holder. A white X-ray pulse with a photon flux of $1 \times 10^{9}$ photons per pulse is produced in the energy range at a $1 \mathrm{kHz}$ repetition rate with the XPS. When multilayer optics with $1 \%$ and $5 \%$ energy bandwidths are used downstream of the XPS, the photon fluxes are $6 \times 10^{7}$ and $3 \times 10^{8}$ photons per pulse, respectively. We can use the discretionary wavelengths and bandwidth in the X-rays for spectra, which is an advantage for the scattering curve corresponding to the asymmetric undulator spectra.

\section{Time-resolved solution scattering of $\mathrm{CH}_{2} \mathbf{I}_{2}$}

Photo-induced chemical and biological reactions have been extensively studied by time-resolved spectroscopic techniques and theoretical calculations. Time-resolved X-ray solution scattering makes it possible to probe transient molecular structures in the photo-induced reactions. We measured the time-resolved scattering signals for photodissociation of the iodine atom form $\mathrm{CH}_{2} \mathrm{I}_{2}$ in methanol (Davidsson et al., 2005).

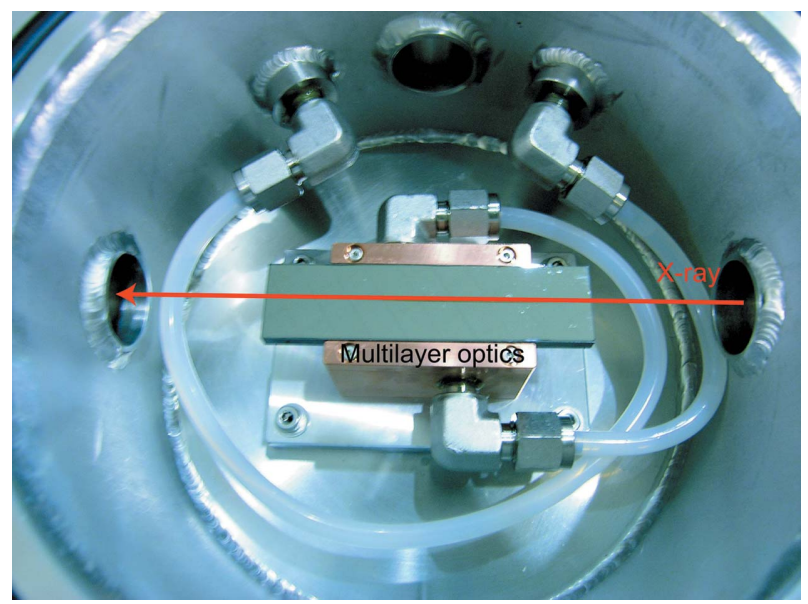

Figure 4

The depth-graded $\mathrm{Ru} / \mathrm{C}$ multilayer in the vacuum chamber installed at the NW14A beamline at the Photon Factory Advanced Ring at KEK. The multilayer is mounted on a water-cooled holder.

We performed the measurement using X-rays with 5\% energy bandwidth at $18 \mathrm{keV}$ to evaluate the feasibility of this set-up.

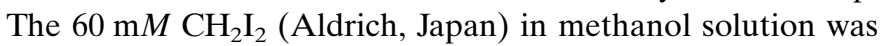
flowed using a liquid jet of thickness $0.3 \mathrm{~mm}$ at a flow rate of about $3 \mathrm{~m} \mathrm{~s}^{-1}$. The open jet makes it possible to remove any background signal owing to the scattering of a glass capillary. The $\mathrm{CH}_{2} \mathrm{I}_{2}$ in methanol solution was excited by $267 \mathrm{~nm}$ light, the third harmonic of the Ti:sapphire femtosecond laser system. To ensure one-photon absorption, the laser pulse width was stretched to $\sim 2$ ps by passing $150 \mathrm{fs}$ laser pulses through a fused silica glass rod cut at the Brewster angle for $267 \mathrm{~nm}$ with $175 \mathrm{~mm}$ optical length. The spot size of both the $\mathrm{X}$-ray and laser beams on the sample surface was $200 \mu \mathrm{m}$ diameter. The laser path was set almost parallel to the X-ray path $\left(\sim 10^{\circ}\right.$ tilt $)$, and the intensity of the laser beam on the sample surface was adjusted to $\sim 35 \mu \mathrm{J}$ per pulse. The sampleto-CCD distance and the exposure time were $48.6 \mathrm{~mm}$ and $7 \mathrm{~s}$ per image, respectively. The CCD detector allowed a $2 \theta$ angle range from about 3 to $62^{\circ}$ to be measured. Difference diffraction data ware measured at time delays of $-200 \mathrm{ps}$, $100 \mathrm{ps}, 300 \mathrm{ps}, 1 \mathrm{~ns}, 3 \mathrm{~ns}, 10 \mathrm{~ns}, 30 \mathrm{~ns}, 50 \mathrm{~ns}, 100 \mathrm{~ns}, 300 \mathrm{~ns}$ and $1 \mu \mathrm{s}$, as shown in Fig. 5. The CCD images were converted to one-dimensional curves using the FIT2D program (http:// www. esrf.eu/computing/scientific/FIT2D/). To extract the diffraction intensity change alone, the data for an unperturbed sample at $-3 \mathrm{~ns}$ were subtracted from the diffraction data collected at other time delays. Photo-induced heating of the solvent is evident in the low $q$ region $\left(\leq 2 \AA^{-1}\right)$. The change in the high $q$ region indicates the photo-induced structural changes of the $\mathrm{CH}_{2} \mathrm{I}_{2}$ molecule. Details of the data analysis will be reported elsewhere.

\section{Conclusion}

Wide-bandwidth X-ray pulses were generated from depthgraded $\mathrm{Ru} / \mathrm{C}$ and $\mathrm{W} / \mathrm{B}_{4} \mathrm{C}$ multilayer optics for time-resolved $\mathrm{X}$-ray solution scattering. The symmetric shape and the 


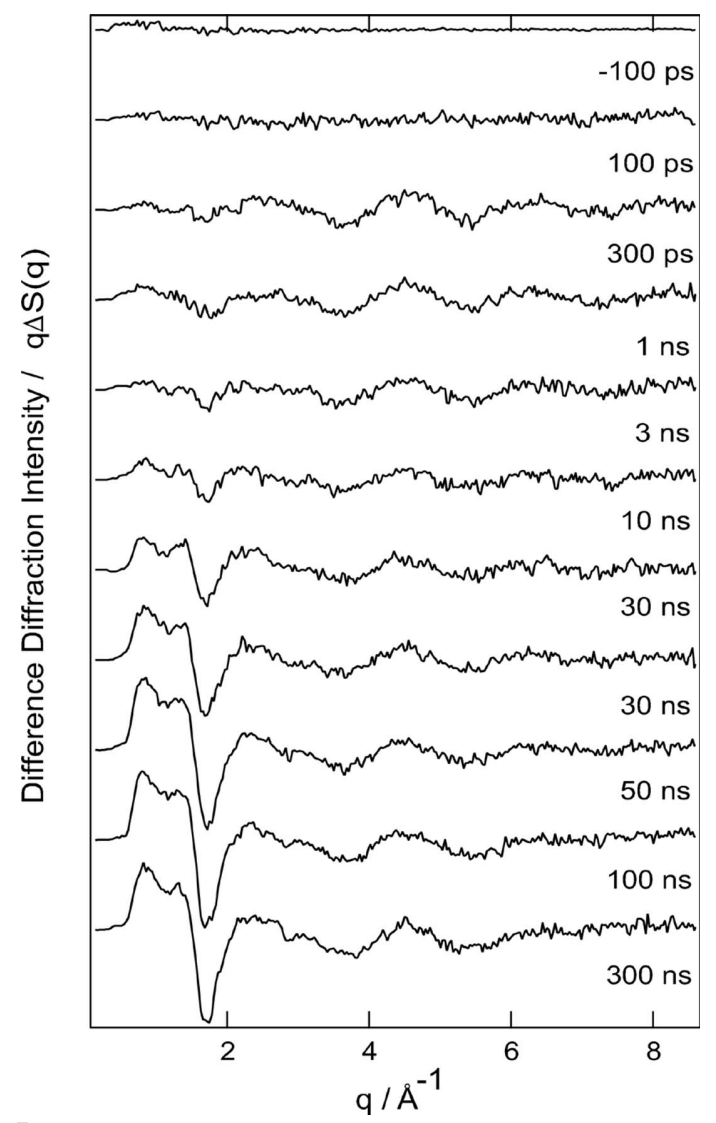

Figure 5

Subnanosecond time-resolved diffraction signal of $\mathrm{CH}_{2} \mathrm{I}_{2}$ in methanol solution as a function of time delay. The differential diffraction intensity was obtained by subtracting the diffraction signal at a reference negative time delay $(-3 \mathrm{~ns})$ from the diffraction signal at each time delay.

bandwidth $(\Delta E / E=1-5 \%)$ of the energy spectra of the X-ray pulse are suitable for time-resolved solution-scattering experiments, and quantitative analysis of photo-induced molecular reaction dynamics in solution. We successfully measured the solution scattering from $\mathrm{CH}_{2} \mathrm{I}_{2}$ in methanol and the time dependence of the difference scattering was presented.

We are grateful to Michael Wulff (ESRF), Atsuo Iida and Hiroshi Kawata (Photon factory, KEK) for discussions of the multilayer optics. We also thank Philip Coppens and Milan Gembicky (State University of New York at Baffalo) for the use of the heat-load chopper. This work was performed under the approval of the Photon Factory Advisory Committee (Proposal No. 2004S1-001) and supported by National Creative Research Initiatives (Center for Time-Resolved Diffraction) of MEST/KOSEF.

\section{References}

Cammarata, M., Lorec, M., Kim, T. K., Lee, J. H., Kong, Q. Y., Pontecorvo, E., Russo, M., Schiró, G., Cupane, A., Wulff, M. \& Ihee, H. (2006). J. Chem. Phys. 124, 124504.

Davidsson, J., Poulsen, J., Cammarata, M., Georgiou, P., Wouts, R., Katona, G., Jacobson, F., Plech, A., Wulff, M., Nyman, G. \& Neutze, R. (2005). Phys. Rev. Lett. 94, 245503.

Gembicky, M., Adachi, S. \& Coppens, P. (2007). J. Synchrotron Rad. 14, 295-296.

Georgiou, P., Vincent, J., Anderson, M., Annemarie, W. B., Gourdon, P., Poulsen, J., Davidsson, J. \& Neutze, R. (2006). J. Chem. Phys. 124, 234507.

Ihee, H. (2009). Acc. Chem. Res. 42, 356-366.

Ihee, H., Lorenc, M., Kim, T. K., Kong, Q. Y., Cammarata, M., Lee, J. H., Bratos, S. \& Wulff, M. (2005). Science, 209, 1223-1227.

Kim, T. K., Lorenc, M., Lee, J. H., Russo, M., Kim, J., Cammarata, M., Kong, Q., Noel, S., Plech, A., Wulf, M. \& Ihee, H. (2006). Proc. Natl. Acad. Sci. USA, 103, 9410-9415.

Kong, Q., Lee, J. H., Plech, A., Wulff, M., Ihee, H. \& Koch, M. H. J. (2008). Angew. Chem. Int. Ed. 47, 5550-5553.

Kong, Q., Wulff, M., Lee, J. H., Bratos, S. \& Ihee, H. (2007). J. Am. Chem. Soc. 127, 13584-13590.

Lee, J. H., Kim, H. K., Kim, T. K., Lee, Y. \& Ihee, H. (2006). J. Chem. Phys. 125, 172504.

Lee, J. H., Kim, J., Cammarata, M., Kong, Q., Kim, K. H., Choi, J., Kim, T. K., Wulff, M. \& Ihee, H. (2008a). Angew. Chem. Int. Ed. 47, 1047-1050.

Lee, J. H., Kim, T. K., Kim, J., Kong, Q., Cammarata, M., Lorenc, M., Wulff, M. \& Ihee, H. (2008b). J. Am. Chem. Soc. 130, 5834-5835.

Mirloup, F., Vuilleumier, R., Bratos, S., Wulff, M. \& Plech, A. (2004). Femtochemistry and Femtobiology, edited by M. Martin and J. T. Hynes. New York: Wiley.

Nozawa, S. et al. (2007). J. Synchrotron Rad. 14, 313-319.

Plech, A., Randler, R., Geis, A. \& Wulff, M. (2002). J. Synchrotron Rad. 9, 287-292.

Plech, A., Wulff, M., Bratos, S., Muirloup, F., Vuilleumier, R., Schotte, F. \& Anfinrud, P. A. (2004). Phys. Rev. Lett. 92, 125505.

Wulff, M., Bratos, S., Plech, A., Vuilleumier, R., Mirloup, F., Lorenc, M., Kong, Q. Y. \& Ihee, H. (2006). J. Chem. Phys. 124, 034501.

Wulff, M., Lorenc, M., Plech, A., Ihee, H., Bratos, S., Mirloup, F. \& Vuilleumier, F. (2004). Femtosecond and Femtobiology, edited by M. Martin and J. T. Hynes. New York: Wiley. 